\title{
Evaluation of Introduction of Simulation-Based Training to Interns
}

\author{
Rajamani Jayadharmarajan Balamurugan ${ }^{1}$, Chandrasekaran Madhubala ${ }^{2}$ \\ ${ }^{1}$ Associate Professor, Department of Anaesthesiology, Government Vellore Medical College Hospital, \\ Adukkamparai, Vellore, Tamilnadu, India. ${ }^{2}$ Postgraduate Student, Department of Anaesthesiology, Government \\ Vellore Medical College Hospital, Adukkamparai, Vellore, Tamilnadu, India.
}

\section{ABSTRACT}

\section{BACKGROUND}

Interns lack practical skills and knowledge regarding emergency lifesaving procedures, especially endotracheal intubation. Even though it is a lifesaving procedure, interns are trained only during their posting in anaesthesia or emergency medicine and so, when need arises, they fail to perform endotracheal intubation, and this leads to catastrophes. We wanted to evaluate the knowledge and skills of endotracheal intubation gained by interns through simulation-based training.

\section{METHODS}

Hundred consenting interns were recruited for the study after obtaining approval from the Institutional Ethics Committee. Mannequin was procured, peers were sensitized, and literature was searched for standard protocol for endotracheal intubation. Feedback form was framed for all stakeholders. IRB approval was obtained, and faculty training was done. After a pre-test, a small sensitization was given regarding endotracheal intubation. Mannequin demonstration was then done by the faculty and doubts were clarified. Then the interns were asked to perform the intubation and post-test was given. Feedback was collected from the students as well as from faculty. Data was compiled and analysed.

\section{RESULTS}

Interns showed mean marks of $6.98 \pm 1.43$ in pre-test compared to $13.19 \pm 0.741$ out of 15 marks post-test with ( $\mathrm{p}=0.0001$ ), which is highly significant. $80 \%$ of interns were satisfied with simulation-based teaching. $75 \%$ of interns were able to perform the procedure in the $1^{\text {st }}$ attempt.

\section{CONCLUSIONS}

The training on mannequin developed competency, basic knowledge, and practice of endotracheal intubation in interns adequately. Training should be carried out at the beginning of internship when they start internship and also in rotation of emergency department. So, they gain competency that is required. In due course, morbidity and mortality due to airway related consequences will reduce if all interns are given hands-on training for intubation on mannequin.

\section{KEY WORDS}

Competency, Direct Observation of Procedural Skills, Endotracheal Intubation, Interns

\section{Corresponding Author:}

Rajamani Jayadharmarajan Balamurugan, Department of Anaesthesiology,

Government Vellore Medical College

Hospital, Adukkamparai, Vellore-632011, Tamilnadu, India.

E-mail: balamuragan75@gmail.com

\section{DOI: $10.14260 /$ jemds/2019/580}

Financial or Other Competing Interests. None.

How to Cite This Article: Balamurugan RJ, Madhubala C. Evaluation of introduction of simulation-based training to interns. J. Evolution Med. Dent. Sci. 2019;8(34):2667-2671, DOI: $10.14260 / \mathrm{jemds} / 2019 / 580$

Submission 05-07-2019,

Peer Review 13-08-2019,

Acceptance 19-08-2019,

Published 26-08-2019. 


\section{BACKGROUND}

Interns lack practical skills and knowledge regarding emergency lifesaving procedures, especially endotracheal intubation. Airway management is one of the most important steps and it is the core content of any emergency medicine training program. Existing undergraduates need training to perform endotracheal intubation with mannequin as well as improve their learning experience with simulation-based training by faculties.

Endotracheal intubation is a lifesaving procedure in emergency or critical situations. Even during internship, it is taught only in Anaesthesia and Emergency Medicine Department, so when any indication emerges, resident cannot perform intubation properly and may lose the patient. When resuscitation is done by non-anaesthetic or nonemergency residents, for endotracheal intubation, they call or depend on anaesthesia or emergency medicine residents and wait till they come, but many a times, the patient's condition cannot wait and leads to catastrophes.

Competency-based education is learner-centred and helps interns for developing competency for endotracheal intubation. This will help them in future to do so whenever the need arises, and a patient's life can be saved.

Simulation based training is one of the most important tools. Simulation ${ }^{1}$ is a technique to amplify real-patient experiences, artificially contrived, that evokes or replicates substantial aspects of the real world in a fully interactive manner ${ }^{1}$. Simulation-based medical education enables knowledge, skills and attitudes to be acquired by health-care professionals in a safe, educationally orientated and efficient manner. ${ }^{2}$

Simulation has been widely accepted as a training and assessment tool in medical education. In the past, trainees relied on the apprenticeship model and routine exposure to patient during their working day to gain expertise in managing such events. Factors contributing to this changing scenario are the changing patterns in health care delivery system such as shorter hospital stays and clinic visits resulting in reduced patient availability for learning, reduced exposure to critically ill patients, limitation on trainee working hours and decreased time for faculty to teach. ${ }^{3}$

Increased patient awareness, improved patient safety and medical litigation have resulted in the increasing use of simulator-based technology into training and assessment programmes in medical education accordingly, the present study was planned to develop competency in interns for endotracheal intubation and to assess that competence achieved. ${ }^{4}$

Skill of a successful endotracheal intubation needs to be acquired by training and attaining several competencies simultaneously. It becomes more challenging when we have to deliver the key concepts in a limited period of time. The medium fidelity simulator is a valuable tool of training for such scenarios. For this purpose, we aim to evaluate the -

1. Knowledge of endotracheal intubation gained by interns through simulation-based training by faculties.

2. The skill of endotracheal intubation- The attempts taken by interns for successful intubation.

3. The satisfaction of faculty in simulation-based training.

4. The satisfaction of interns in simulation-based training.

\section{METHODS}

This study conducted in the department of Anaesthesia for interns. A group of 100 students were taught about intubation in mannequin using a power point, video and demonstration. Doubts were cleared then and there. Assessment of knowledge (Cognition) by giving pre and post-test. Questionnaire of 15 questions each carrying one mark was framed using standard protocol for this purpose.

\section{Study Centre}

The study was conducted in Government Vellore Medical College and Hospital, Adukamparai, Vellore. We selected interns posted in general surgery, General Medicine, OG and Community Medicine department during their free time.

\section{Study Design}

This was a observational study.

\section{Sampling Method}

Convenient sampling.

\section{Sample Size}

100.

\section{Sample Population}

Interns in government Vellore medical college.

\section{Study Period}

3 months.

\section{Study Area}

Government Vellore Medical College, Vellore, Tamilnadu.

\section{Methods of Data Collection}

This study conducted in the department of Anaesthesia for interns a pre-test was conducted using a validated questionnaire consisting of 15 short answer questions. This questionnaire was prepared with suggestions from three expert faculty members of the Department of Anaesthesiology. All interns attended didactic sessions reviewing airway anatomy, general principles of airway management, details of equipment such as laryngoscope, endotracheal tube and bagmask resuscitator and procedure for laryngoscope and endotracheal intubation (L and ETI). They watched a short video illustrating laryngoscope and observed a demonstration of the procedure on a mannequin by the faculty. Subsequently the interns were asked to perform the skill of intubation in the mannequin (Psychomotor skills assessed). The same questionnaire given previously was circulated, and the interns were evaluated using a post-test (Cognition assessed).

\section{Statistical Analysis}

The data was analysed using SPSS and Microsoft excels 2010. Paired $t$ test was used to compare the means of a dependent variables between two groups. These included the mean and standard Deviation (SD) for quantitative variables. The statistically significant $p$ value was set at $<0.0001$ and the $9.5 \%$ confidence interval. 


\section{RESULTS}

Totally 100 students were participated in our study. There was significant difference noted between the groups with respect to marks obtained. Interns showed mean \& SD in the pre-test was $(6.98 \pm 1.43)$ and post-test was $(13.19 \pm 0.741)$. The difference between two population mean $(p=0.0001)$ statistically significant, which was calculated by paired $t$ test. $80 \%$ of interns satisfied with simulation-based teaching $75 \%$ of interns able to perform the procedure in the $1^{\text {st }}$ attempt. $80 \%$ of interns satisfied with simulation-based teaching $75 \%$ of interns able to perform the procedure in the $1^{\text {st }}$ attempt there was a definite gain in knowledge after the sessions, with highly significant statistical difference. For evaluation by DOPS, interns were given to attempts for all three steps. Attempt was considered as first if they done successfully, and second if failed and done again successfully.

\begin{tabular}{|c|c|c|c|}
\hline \multirow{2}{*}{ Results } & \multicolumn{2}{|c|}{ Marks Obtained (Out OF 15) } & \multirow{2}{*}{ p Value } \\
\cline { 2 - 3 } & Pre-Test & Post-Test & \\
\hline Mean \pm SD & $6.98 \pm 1.43$ & $13.19 \pm 1.0741$ & $<0.0001^{*}$ \\
\hline \multicolumn{3}{|c|}{ Table 1. Pre \& Post Test Results } \\
\hline
\end{tabular}

\begin{tabular}{|c|c|c|c|c|c|c|c|}
\hline \multirow{2}{*}{ Scale } & \multicolumn{7}{|c|}{ Question Number Percentage of Interns } \\
\cline { 2 - 8 } & $\mathbf{Q 1}$ & $\mathbf{Q 2}$ & $\mathbf{Q 3}$ & $\mathbf{Q 4}$ & $\mathbf{Q 5}$ & $\mathbf{Q 6}$ & $\mathbf{Q 7}$ \\
\hline Strongly agree & 86 & 84 & 84 & 76 & 85 & 84 & 82 \\
\hline Agree & 12 & 12 & 12 & 16 & 09 & 14 & 10 \\
\hline Somewhat agree & 02 & 04 & 04 & 08 & 06 & 02 & 08 \\
\hline Disagree & - & - & - & - & - & - & - \\
\hline Strongly disagree & - & - & - & - & - & - & - \\
\hline Total & $\mathbf{1 0 0}$ & $\mathbf{1 0 0}$ & $\mathbf{1 0 0}$ & $\mathbf{1 0 0}$ & $\mathbf{1 0 0}$ & $\mathbf{1 0 0}$ & $\mathbf{1 0 0}$ \\
\hline \multicolumn{8}{|c|}{ Table 2. Feedback Questionnaire Analysis } \\
\hline
\end{tabular}

Q1: Material informative and easy to understand; Q2: Visibility and clarity of presentation; Q3: Instructions given during training is helpful; Q4: Hands on training for each candidate is helpful Q5: confidence to carry out endotracheal intubation after training; Q6: Useful and should be taught during MBBS Q7: whether this mannequin helpful for/ICU and theater procedure.

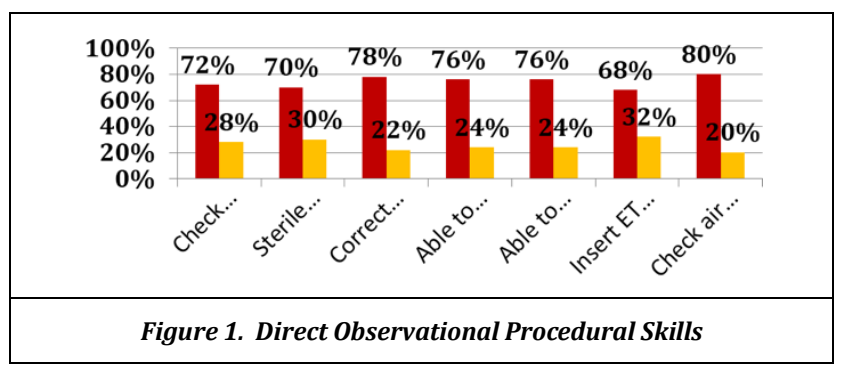

Shows the stepwise results of DOPS. It shows that $72 \%$ of interns check airway equipment. 70\% interns followed sterile techniques. $78 \%$ position the patient correctly. $76 \%$ place handle in left hand and able to lateralize the tongue. $76 \%$ interns able to visualize the vocal cord. $65 \%$ interns able to insert endotracheal tube into vocal cord. $80 \%$ interns check air entry equal on both sides.

Intubation was successful at first attempt in $75 \%$ of interns while $20 \%$ at second and $5 \%$ at third attempts. Ventilation with bag and mask was possible in all the interns $(90 \%$ at first and $10 \%$ on second attempt) as no one required doing it again. In the procedure of endotracheal intubation, ventilation with bag and mask is more important to prevent hypoxia and its consequences such as irreversible brain damage. As all interns had successfully done, it shows their immediate competency for that

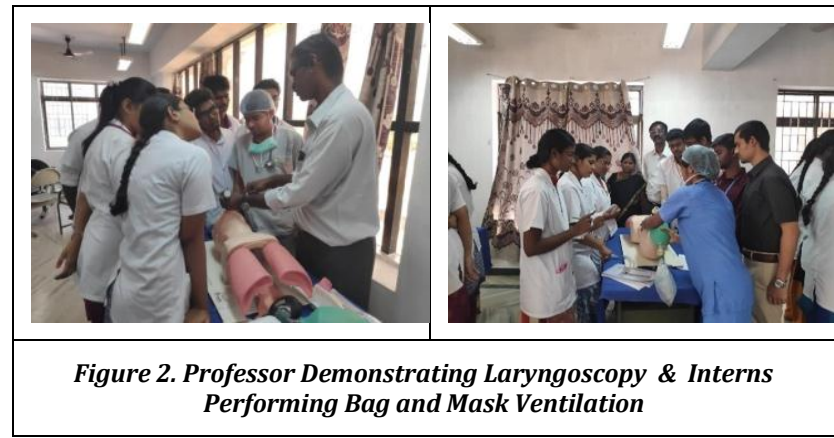

Of the 100 interns, 75 interns able to perform the procedure in the first attempt and 25 interns able to intubate with more attempts and assistance [Figure 3] However, all were able to do it in a single training that means that it is a procedure which can be done by them if they are adequately trained for that.

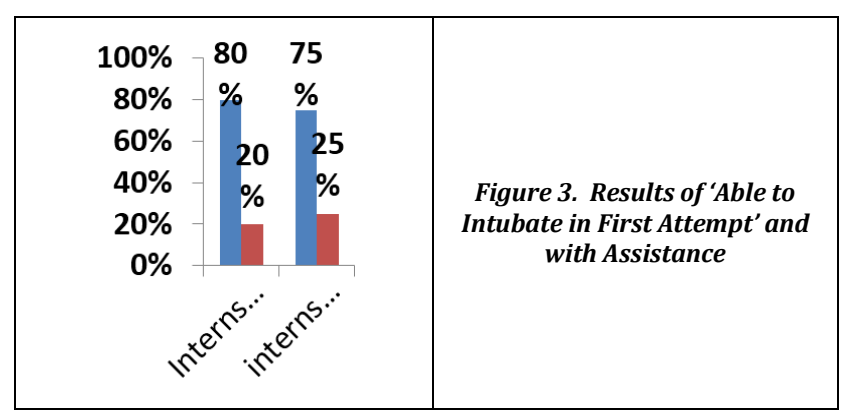

Means that it is a procedure that requires repeat training or exposure to that. However, all were able to do it in a single training that means that it is a procedure which can be done by them if they are adequately trained for that. At the end of the session, evaluation was done using questionnaire with Likert scale from 1 to 5 where 1 denotes strongly agree and 5 denotes strongly disagree. The results show that eighty six percent interns found the material informative and easy to understand. Eighty-four percent interns strongly agreed for visibility and clarity of presentation. Eighty-four interns found that instructions given during training were helpful. Eighty five percent interns strongly agreed for confident to carry out endotracheal intubation in future after hands-on training. Eighty two percent interns found mannequin is helpful for ICU and theatre procedure.

\section{DISCUSSION}

Simulation, in all its variants, is an excellent tool for health care educators as it allows students to achieve these goals without patients being put at risk. The task becomes extremely difficult for the novice trainees in a real-life where they have to perform it on an actual patient with simultaneous attention to cardio-respiratory status. With the current trend in the reduction of medical training hours or short duration of the attachment, this condition may get further compromised. Simulation is a safe nonthreatening5, non-urgent method of 
providing repetitive practice to acquire and achieve proficiency in such skills. Our results showed a definite role of simulation in teaching the technical skills related to tracheal intubation in students who had simulated sessions hence proving it as an effective teaching strategy. The strategy was also helpful in orienting the students with the equipment and theatre environment which is very important for them to perform endotracheal intubation Gaba noted that simulation has the potential to revolutionise health care and address patient safety issues if appropriately utilised and integrated into the educational and organisational improvement process. Trainees can make errors and learn to recognise and correct them in the simulated environment without fear of being penalised or causing harm to patients.

Rosenthal et al. found simulation to be a safe and valid evaluation and training tool for emergency airway management skills of interns ${ }^{6}$. Basic airway management is a major component of resuscitation. The impact of a basic airway skills simulation programme on residents is tremendous with significantly better performance on most elements of basic airway management during mock codes. According to Vision 2015 by the Medical council of India, ${ }^{5}$ all medical graduates should be able to manage the airway during emergency situations. Endotracheal intubation is the most effective method of securing the airway, but it is difficult to be competent without adequate training. Endotracheal intubation is taught in a series of steps to avoid cognitive overload. ${ }^{1}$

Owen and Plummer ${ }^{7}$ trained 100 students and trainees for endotracheal intubation on models (Mannequin) similar to us and found that they were more comfortable learning on models than patients. Salam et al. ${ }^{8}$ Done a cross-sectional study (2003-2005) and assessed competency in interns for endotracheal intubation. They found that even though its knowledge is compulsory during major clinical posting, out of $70 \%$ of interns, only $40 \%$ claimed about their competency of endotracheal intubation. They suggested the curriculum setter to think about core competency to be developed at house officer level. In our study, interns were previously not exposed to endotracheal intubation, but still shown better.

Bernhard et $\mathrm{al}^{9}$ (2007-2010) done a study on 21 residents for $1^{\text {st }}$ year in anaesthesia to perform 200 endotracheal intubation and assessed for associated success rate and number of attempts needed until successful in consecutive blocks of 25 procedures. They found a success rate of $67 \%$ in the first attempt compared to $83 \%$ after 200 number of intubations. Their overall success rate was $82 \%$ similar to $84 \%$ of our study. They also found that number of attempts for intubations were required less after the first 25 intubations (1.6 \pm 0.8$)$ compared to $1.3 \pm 0.6$ after 200 intubations ( $\mathrm{P}=$ 0.0001 ). They have not mentioned about the technique and time period of training before evaluation, but our success rate is high compared to them in the first training, which shows efficacy of our training.

Kusel et al. ${ }^{10}$ (2010-2012) studied the factors affecting the outcome of interns during anaesthesia rotation for a period of 3 years. They observed that $80 \%$ were able to perform intubation and more than $80 \%$ were confident about intubation. Their result was similar to ours study. They also found that for more than $80 \%$ success rate, they required 29 procedures to be performed by interns indicating that repetition of practice or simulation should be done after some time for retention of skills. Dehmer et al did online survey in $1564^{\text {th }}$ year medical students for endotracheal intubation and found that out of 134 students, 83 never performed endotracheal intubation and more than 118 were not confident for it at all or without major assistance.

Mayo et al.11 found poor performance for airway management in Advanced Cardiac Life Support (ACLS) certified interns at beginning of internship, which was found excellent after training as compared to beginning of internship. Katowa-Mukwato et al, 12 had conducted crosssectional survey among $5^{\text {th }}, 6^{\text {th }}$, and $7^{\text {th }}$ year medical students, 2 months before the final examination and found that 19 students never performed endotracheal intubation and not confident for that. Eighteen students performed it only once and 12 students performed endotracheal intubation more than two times and they were confident for it. They found high correlation between experience and confidence. The student who had performed the procedure had improved rate of selfconfidence. They were not allowed to perform endotracheal intubation frequently in emergency, so confidence level was low which would be required high for emergency management. They recommended simulation practice for lifesaving procedures such as endotracheal intubation to increase their confidence level, which we have done in our study. Burch et al. ${ }^{13}$ assessed 58 graduates of South African medical schools and found an average score of $67 \%$ for success compared to $75 \%$ of our study for endotracheal intubation. They concluded that most of the students were unable to perform it on entry to preregistration year and required further training.

Despite efforts from educators to set a more independent and self-directed learning approach and curriculum, a disconnect still exists between the classroom and the clinical environment. Medical simulation has been proposed as a technique to bridge this educational gap.

The results of this study suggest that simulation-based teaching is more effective than the traditional apprenticeshipbased model in teaching airway management skills. Simulation has considerable application in testing airway devices as well as teaching procedural competence. All health-care practitioners should embrace simulation as a tool to aid teaching and learning across a wide range of cognitive, behavioural and psychomotor competence knowledge. The use of simulation-based assessment and retraining is a promising and innovative tool that deserves further exploration.

\section{CONCLUSIONS}

Training for endotracheal intubation should be carried out at the beginning of internship and can be repeated during their posting in Anaesthesia and Emergency Medicine Departments. The training on mannequin improved their competency, basic knowledge, and practice of endotracheal intubation in interns adequately. In due course morbidity and mortality due to 
airway related conditions will reduce if all interns are given hands on training for intubation on mannequin.

\section{ACKNOWLEDGEMENT}

We are thankful to Dr. Shantimalar, the Dean of our Medical College, and Dr. S. Rajavelu, The Medical Superintendent of our hospital, for allowing us and also helping us in completing this study. We are also very thankful to Dr. Gomathi, Dr. Senthilkumar, and all the staff from the Department of Anaesthesia.

\section{REFERENCES}

[1] Gaba DM, DeAnda A. A comprehensive anesthesia simulation environment: re-creating the operating room for research and training. Anesthesiology 1988;69(3):387-94.

[2] Satava RM. Virtual reality surgical simulator. The first steps. Surg Endosc 1993;7(3):203-5.

[3] Bradley P. The history of simulation in medical education and possible future directions. Med Educ 2006;40(3):254-62.

[4] Peterson GN, Domino KB, Caplan RA, et al. Management of the difficult airway: a closed claims analysis. Anesthesiology 2005;103(1):33-9.

[5] Higham H, Baxendale B. Evidence supporting simulationbased training. Simulation ement/MCI_booklet.pdf training is an effective educational modality, with strong evidence demonstrating improvement in learners' knowledge, skills and behaviors.... use of simulation to enhance training and patient safety in anaesthesia. Br J Anaesth 2017;119(Suppl 1):i106-i14.

[6] Rothstein J, Gullo J, Cuningham L, et al. Training emergency medicine residents- the skill of endotracheal intubation: comparison of human cadavers vs mannequins. Panamerican J Trauma Crit Care Emerg Surg 2014;3(1):8-10

[7] Owen H, Plummer JL. Improving learning of a clinical skill: the first year's experience of teaching endotracheal intubation in a clinical simulation facility. Med Educ 2002;36(7):635-42.

[8] Salam A, Md. Zainuddin Z, Latiff AA, et al. Assessment of medical graduates' competencies. Ann of the Academy of Medicine Singapore 2008;37(9):814-6.

[9] Bernhard M, Mohr S, Weigand MA, et al. Developing the skill of endotracheal intubation: implication for emergency medicine. Acta Anaesthesiol Scand 2012;56(2):164-71.

[10] Kusel B, Farina Z, Aldous C. Anaesthesia training for interns at a metropolitan training complex: Does it make the grade? S Afr Fam Pract 2014;56(3):201-5.

[11] Mayo PH, Hackney JE, Mueck JT, et al. Achieving house staff competence in emergency airway management: Results of a teaching program using a computerized patient simulator. Crit Care Med 2004;32(12):2422-7.

[12] Katowa-Mukwato P, Andrews B, Maimbolwa M, et al. Medical students' clerkship experiences and selfperceived competence in clinical skills. Afr J Health Prof Educ 2014;6(2):155-60.

[13] Burch VC, Nash RC, Zabow T, et al. A structured assessment of newly qualified medical graduates. Medical Education 2005;39(7):723-31. 